\title{
Local inflammation enables a basophil-neuronal circuITCH in atopic dermatitis
}

\author{
Anna Ehlers ${ }^{1}$, Celia López Sanz ${ }^{2}$, and Rodrigo Jimenéz-Saiz ${ }^{2}$ \\ ${ }^{1}$ Universitair Medisch Centrum Utrecht \\ ${ }^{2}$ Hospital Universitario de la Princesa
}

June 25, 2021

\begin{abstract}
Acute itch flares in atopic dermatitis via the LTC4-CysLTR2 pathway.

Title : Local inflammation enables a basophil-neuronalcircuITCH in atopic dermatitis

Authors: Celia López-Sanz ${ }^{1}$, Rodrigo Jimenéz-Saiz ${ }^{1-4}$, Anna M Ehlers ${ }^{5,6}$

${ }^{1}$ Department of Immunology, Instituto de Investigación Sanitaria Hospital Universitario de La Princesa (IIS-IP), Madrid, Spain

${ }^{2}$ Department of Immunology and Oncology, Centro Nacional de Biotecnología (CNB)-CSIC, Madrid, Spain

${ }^{3}$ Faculty of Experimental Sciences, Universidad Francisco de Vitoria (UFV), Madrid, Spain

${ }^{4}$ McMaster Immunology Research Centre, Department of Medicine, McMaster University, Hamilton, Ontario, Canada

${ }^{5}$ Center for Translational Immunology, University Medical Center Utrecht, Utrecht University, Utrecht, The Netherlands

${ }^{6}$ Department of Dermatology/Allergology, University Medical Center Utrecht, Utrecht University, Utrecht, The Netherlands
\end{abstract}

Correspondence to:

Anna M Ehlers

Center for Translational Immunology

Heidelberglaan 100,

3584 CX Utrecht, The Netherlands

a.m.ehlers-2@umcutrecht.nl

Keywords: basophils; allergic inflammation; leukotriene C; neuroimmunology; atopic dermatitis; itch;

Abbreviations: AD, atopic dermatitis; CCL, C-C chemokine ligand; CCR; C-C chemokine receptor; CysLTR, Cysteinyl leukotriene receptor; DT, diphtheria toxin; HR, histamine receptor; LT, leukotriene; MCs, mast cells; OVA, ovalbumin.

Main text 
Allergic inflammation is often the result of a dysregulated Th2 immune response, IgE production, and the release of allergic mediators such as histamine or leukotrienes (LTs) by basophils and mast cells (MCs). Allergic diseases can manifest as acute allergic reactions (anaphylaxis), or as chronic allergic inflammation in chronic urticaria, allergic rhinitis, allergic asthma, and atopic dermatitis (AD). ${ }^{1}$ Common allergic symptoms such as sneezing, airway mucus secretion, and chronic itch are caused by interactions between immune cells and sensory neurons in the inflamed tissue. ${ }^{2,3}$

The MC-neuronal axis is involved in chronic itch experienced by AD patients. Stimulated MCs release, among other mediators, histamine, which directly activates pruriceptor sensory neurons via histamine receptors (HR) and elicit itch. ${ }^{3}$ However, it remains unclear how acute itch flares are triggered in certain subpopulations of AD patients. Recently, Wang et al. observed that acute itch flares were associated with increased serum specific IgE in patients with moderate to severe AD. ${ }^{4}$ They showed MC-independent acute itch flares in an AD-like disease murine model sensitized to ovalbumin (OVA) and deficient in MCs ( $\mathrm{Sash}^{-/}$mice) upon OVA challenge. Additionally, they performed basophil depletion experiments using anti-CD200R in wild type mice, and diphtheria toxin (DT) in Bas-TRECK mice, which exclusively express the DT receptor on basophils. Altogether, Wang et al . uncovered the requirement of basophils for acute itch flares, and its redundancy for chronic itch behavior and specific $\operatorname{IgE}$ production in $\mathrm{AD}$.

The critical role of basophils in acute itch flares was restricted to chronic AD-like skin disease because acute itch flares in OVA-sensitized wild type mice were MC- but not basophil-dependent. These data were strengthened by passive immunization experiments with OVA-specific IgE, where acute itch flares were only basophil-dependent in the $\mathrm{AD}$-associated inflammatory disease model. ${ }^{4}$ This apparent riddle was explained by the scarce dermis infiltration of circulating basophils without previous inflammation. In contrast, achronically-inflamed environment promoted basophil recruitment that enabled them to migrate through the dermis to interact with sensory neurons. This interaction caused acute pruritus via the LTC4-Cysteinyl leukotriene receptor (CysLTR) 2 pathway (Fig. 1 ). ${ }^{4}$

LTs are well-known lipid mediators involved in several pro-inflammatory responses via CysLTR1 or CyLTR2, which are expressed on murine and human dorsal root ganglia neurons. ${ }^{4}$ Besides its involvement in AD-associated acute itch flares, the LTC4-CysLTR2 pathway was also required for MC activation and eosinophil-dependent skin fibrosis in an OVA-induced AD murine model..$^{5}$ Interestingly, recent studies have demonstrated the relevance of the LTC4-CysLTR2 axis for acute and chronic itch behavior in chronic inflammation. On the other hand, this axis was redundant for acute (Alternaria -induced itch) or non-inflammatory itch (dry skin induced itch). ${ }^{5}$

Chronic tissue inflammation promotes basophil infiltration via C-C chemokine receptor (CCR) 1,2 and 3 that bind to chemoattractants such as C-C chemokine ligand (CCL) 2, 5 and 11. CCR-CCL interactions upregulate $\alpha$ - and $\beta$-integrin in basophils and other leukocytes. These integrins interact with vascular cell adhesion molecules, fibronectin or intercellular adhesion molecules 1-3 of the vascular endothelium for diapedesis. ${ }^{6}$ In addition, the release of damage-associated molecular patterns, or alarmins, such as thymic stromal lymphopoietin, IL-33 and IL-25, empowers basophil recruitment into the inflamed tissue. Also, allergens such as Der $\mathrm{f} 2$ fromDermatophagoides farinae have been reported to induce, and even enhance, chemokine-induced basophil migration. ${ }^{7}$ It has been shown by 2 -photon imaging of (murine) skin lesions and airway histology of fatal asthma cases that, alike MCs, tissue-infiltrated basophils can locate near to sensory neurons, ${ }^{4,8}$ which likely favors a basophil-neuronal interplay.

Basophils were long thought to be redundant with MCs in atopy, until recently, that their role has been recognized in different phases of allergic pathology, ${ }^{1}$ including sensitization and anaphylaxis. ${ }^{9}$ The newly identified basophil-dependent LTC4-CysLTR2 pathway may contribute to chronic allergic diseases other than AD. For example, allergic rhinitis and allergic asthma are characterized by recurrent (on-season) allergen exposure, which may trigger continuous basophil tissue infiltration (Fig. 1 ). Also, it would be intriguing to examine whether subclinical doses of food allergens, which can be constantly ingested without symptomatology, can promote basophil migration to the oro-gastrointestinal tract. This may be particularly relevant for oral immunotherapy, characterized by the administration of steadily increasing doses of allergen over 
months, and even years, for desensitization. Thus, targeting these putative basophil-neuronal interactions during oral immunotherapy may improve patient's safety and compliance by reducing adverse side events.

In conclusion, the work by Wang et al. adds insight on basophil-neuronal interactions to the brand new and rapidly growing field of neuroimmunology. Understanding the fundamental mechanisms and immunological principles that govern effector cell (MC/basophil)-neuron communication in the landscape of chronic allergic inflammatory diseases is a prerequisite for the identification of novel therapeutic targets in allergy.

Conflict of interest : The authors have no conflict of interest to declare.

Financial support : RJS's laboratory acknowledges the support received by the Severo Ochoa Program (AEI/SEV-2017-0712), FSE/FEDER through the Instituto de Salud Carlos III (ISCIII; CP20/00043), The Nutricia Research Foundation (NRF-2021-13), New Frontiers in Research Fund (NFRFE-2019-00083), and SEAIC (BECA20A9).

Authorship : The authors approved the final version of the manuscript as submitted and agreed to be accountable for all aspects of the work.

Acknowledgements : The authors want to thank Dr. Anna Globinska for graphical abstract design. CLS and AME drafted the manuscript and RJS provided critical feedback and revisions. All authors approved the final version for submission.

\section{References}

1. Miyake, K. and H. Karasuyama, Emerging roles of basophils in allergic inflammation. Allergol Int, 2017. 66 (3): p. 382-91.

2. Voisin, T., A. Bouvier, and I.M. Chiu, Neuro-immune interactions in allergic diseases: Novel targets for therapeutics. Int Immunol, 2017. 29 (6): p. 247-61.

3. Kabata, H. and D. Artis, Neuro-immune crosstalk and allergic inflammation. J Clin Invest, 2019. 129 (4): p. $1475-82$.

4. Wang, F., et al., A basophil-neuronal axis promotes itch.Cell, 2021. 184 (2): p. 422-40.e17.

5. Voisin, T., et al., The CysLT2R receptor mediates leukotriene C4-driven acute and chronic itch. Proc Natl Acad Sci U S A, 2021.118 (13): p. e2022087118.

6. Korosec, P., et al., Basophils, high-affinity IgE receptors, and CCL2 in human anaphylaxis. J Allergy Clin Immunol, 2017.140 (3): p. 750-8.e15.

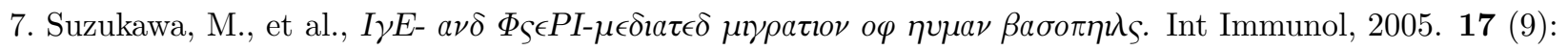
p. $1249-55$.

8. Koshino, T., et al., Identification of basophils by immunohistochemistry in the airways of post-mortem cases of fatal asthma. Clin Exp Allergy, 1993. 23 (11): p. 919-25.

9. Kashiwakura, J.-I., et al., The basophil-IL-4-mast cell axis is required for food allergy. Allergy, 2019. 74 (10): p. 1992-6.

\section{Figure Legend}

Figure 1. Acute itch flares in AD patients are associated with increased levels of specific IgE (top left side). While chronic itch behavior is dependent on MC-neuronal communication, acute itch relies on basophilneuronal interaction via the LTC4-CysLTR2 pathway (bottom), as demonstrated with different AD-like disease murine models sensitized to OVA (top middle). The underlying requirement for this pathway seems to be chronic inflammation, which can also occur in other allergic diseases. A continuous local inflammation may promote basophil tissue infiltration, where they can reside in proximity to sensory neurons favoring a basophil-neuronal interplay (right side). Bas, basophils. 


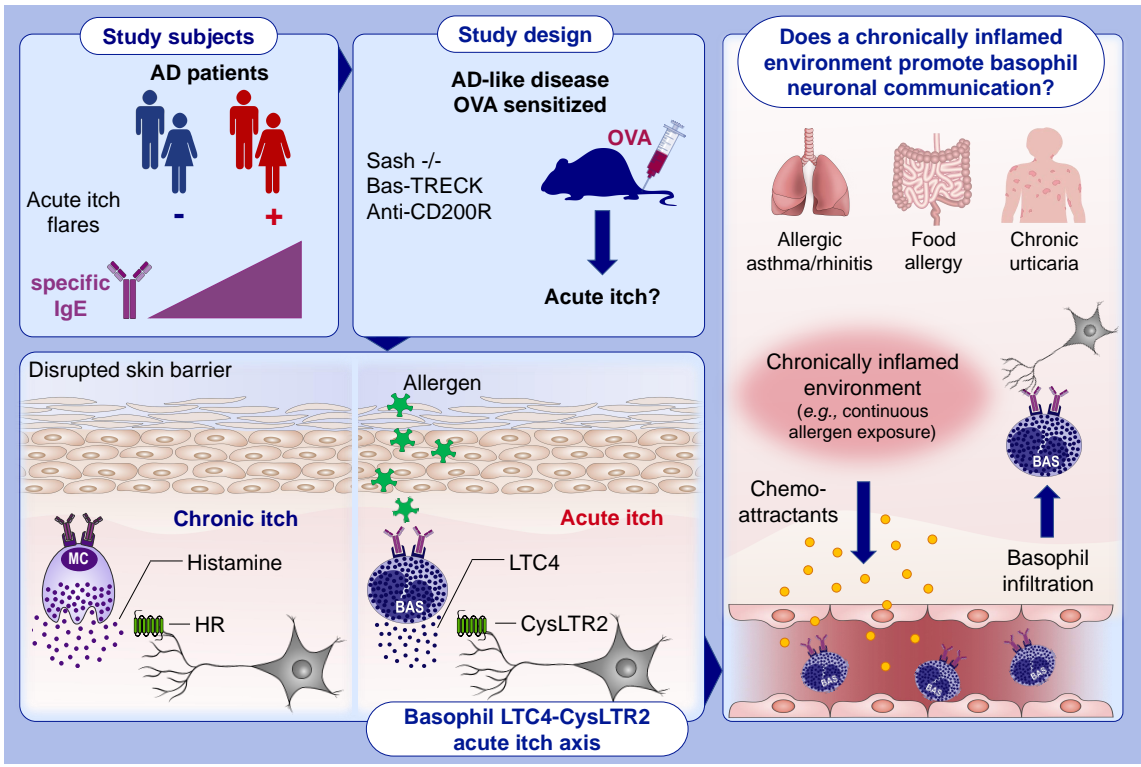

